\section{The structural basis of R-spondin recognition by LGR5 and RNF43}

\author{
Po-Han Chen, ${ }^{1}$ Xiaoyan Chen, ${ }^{1}$ Zhenghong Lin, ${ }^{2}$ \\ Deyu Fang, ${ }^{2}$ and Xiaolin $\mathrm{He}^{1,3}$ \\ ${ }^{1}$ Department of Molecular Pharmacology and Biological \\ Chemistry, Northwestern University Feinberg School \\ of Medicine, Chicago, Illinois 60611, USA; ${ }^{2}$ Department \\ of Pathology, Northwestern University Feinberg \\ School of Medicine, Chicago, Illinois 60611, USA
}

R-spondins (RSPOs) enhance Wnt signaling, affect stem cell behavior, bind to leucine-rich repeat-containing G-proteincoupled receptors 4-6, (LGR4-6) and the transmembrane E3 ubiquitin ligases RING finger 43/zinc and RING finger 3 (RNF43/ZNRF3). The structure of RSPO1 bound to both LGR5 and RNF43 ectodomains confirms their physical linkage. RSPO1 is sandwiched by LGR5 and RNF43, with its rod module of the cysteine-rich domain (CRD) contacting LGR5 and a hairpin inserted into RNF43. LGR5 does not contact RNF43 but increases the affinity of RSPO1 to RNF43, supporting LGR5 as an engagement receptor and RNF43 as an effector receptor. Disease mutations map to the RSPO1RNF43 interface, which promises therapeutic targeting.

Supplemental material is available for this article.

Received April 14, 2013; revised version accepted May 17, 2013.

Wnt signaling plays crucial roles in development across species. Underlying its evolutionary significance is the large repertoire and dynamic assembly of receptors, coreceptors, agonists, and antagonists involved in Wnt signaling regulation (Clevers and Nusse 2012; Niehrs 2012). The core apparatus comprises the serpentine transmembrane receptor Frizzled $(\mathrm{Fz})$, the coreceptor LRP6 in the case of canonical Wnt $/ \beta$-catenin pathways, and other receptors such as Syndecan and ROR for noncanonical pathways (Wnt/PCP, for instance). Recent revelation of the core WntFz complex structure resolved long-standing questions on the molecular basis of Wnt-Fz recognition (Janda et al. 2012). Beyond Wnt-Fz, much remains unknown about how other regulators assemble to modulate Wnt pathways.

The secreted glycoproteins R-spondins 1-4 (RSPO1-4) were discovered as potent activators of Wnt signaling (Kamata et al. 2004; Kazanskaya et al. 2004; de Lau et al. 2012; Cruciat and Niehrs 2013). Their enigmatic nature is highlighted by their Wnt-dependent synergistic effects, their presence only in vertebrates plus selected invertebrates, and their low sequence homology with other Wnt signaling molecules (de Lau et al. 2012; Cruciat and Niehrs 2013). RSPOs consist of an N-terminal Cys-rich furin-like

[Keywords: Wnt signaling; LGR5; R-spondin; RNF43; furin-like repeat; E3 ubiquitin ligase]

${ }^{3}$ Corresponding author

E-mail x-he@northwestern.edu

Article published online ahead of print. Article and publication date are online at http://www.genesdev.org/cgi/doi/10.1101/gad.219915.113. domain (CRD) necessary and sufficient for activities (Kazanskaya et al. 2004; Glinka et al. 2011), a thrombospondin (TSP) type I repeat domain for heparan sulfate proteoglycan binding (Ohkawara et al. 2011), and a C-terminal basic region of variable lengths. Underlying the critical role of the CRD in signaling, RSPO1 mutations in this region lead to XX sex reversal and skin abnormalities (palmoplantar keratosis) (Parma et al. 2006), and RSPO4 mutations result in developmental defect in fingernails and toenails (anonychia) (Blaydon et al. 2006). The identities of RSPO receptors had been controversial. It was originally suggested that RSPOs bind to Fz or LRP6 (Wei et al. 2007; Ohkawara et al. 2011); in another study, RSPOs were reported to antagonize the Wnt inhibitor Kremen. None of the results from these studies were confirmed, however (Binnerts et al. 2007).

Most recently, several independent studies identified LGR4-6 (leucine-rich repeat [LRR]-containing G-proteincoupled receptors [GPCRs] 4-6) as receptors for RSPO1-4 (Carmon et al. 2011; de Lau et al. 2011; Glinka et al. 2011; Ruffner et al. 2012). LGR4-6 mark adult stem cell compartments in several organ systems and are necessary for proper kidney, mammary, and intestinal development (Barker and Clevers 2010; Snippert et al. 2010; Barker et al. 2012; Huch et al. 2013). LGR4-6 are closely related to GPCRs for glycoprotein hormones FSH (LGR1 as the receptor), LH (LGR2), TSH (LGR3), and relaxins (LGR7 and LGR8) (Hsu et al. 2000; Kong et al. 2010), all of which share a similar architecture: a large $\mathrm{N}$-terminal extracellular LRR domain for ligand binding, a seven-helix transmembrane domain, and a short cytosolic region. Intriguingly, unlike the hormone receptors, LGR4-6 are not known to use $\mathrm{G}$ proteins for signaling, provoking an intriguing question about their role as RSPO receptors.

LGR5-RSPO1 has been reported to form a ternary complex with the transmembrane RING-type E3 ubiquitin ligase ZNRF3 (zinc and RING finger 3) or RNF43 (RING finger 43) (Hao et al. 2012; Koo et al. 2012). This finding suggested a mechanism by which RSPO1 potentiates Wnt signaling. Being a Wnt target gene, ZNRF3/RNF43 balances Wnt signaling by providing a steady-state membrane clearance of the Fz-LRP complex (Hao et al. 2012; Koo et al. 2012). Through interacting with and inducing endocytosis of ZNRF3/RNF43, RSPO1 inhibits ZNRF3/RNF43 activities and henceforth stabilizes the surface level of FzLRP for canonical and noncanonical Wnt signaling (Hao et al. 2012). Importantly, the RSPO1-mediated ZNRF3 membrane clearance is LGR-dependent, which is supported by biochemical data that the three proteins could form a physical complex (Hao et al. 2012).

To clarify the structural roles of RSPOs, LGRs, and ZNRF3/RNF43 in Wnt signaling, we determined the crystal structure of the human ternary LGR5-RSPO1RNF43 complex at $2.5 \AA$ resolution. Our results reveal the RSPO CRD fold, provide direct evidence that RSPOs physically tether LGR4-6 and ZNRF3/RNF43 coreceptors, and provide insights into the roles of LGR4-6 and ZNRF3/RNF43 in the signaling complex assembly.

\section{Results and Discussion}

\section{Assembly of the ternary complex}

The LGR5 ectodomain (ECD), RNF43 protease-associated (PA) domain, and RSPO1 CRD were used for the structural 
studies (Fig. 1A). Because LGR5 aggregates easily by itself, it was coexpressed with RSPO1 in HEK293S cells (Materials and Methods; Supplemental Material), and affinity-purified RSPO1-LGR5 was mixed with RNF43 to reconstitute the ternary complex. The structure was determined by single isomorphous replacement with anomalous scattering (SIRAS) (Supplemental Table 1). Consistent with previous findings, the structure confirms the physical linkage between the three proteins (Hao et al. 2012), with RSPO1 CRD sandwiched between two coreceptors (Fig. 1B). RNF43 and LGR5 C termini point in the same direction, leading to the cell membrane, suggesting that the observed structural assembly mimics the biologically relevant complex.

Overall, the position of RSPO1 recognition by LGR5 is reminiscent of the position of FSH recognition by FSHR at the N-terminal LRR region (Supplemental Fig. 1), despite the fact that RSPO1 and FSH are of different folds. FSHR uses its additional C-terminal hinge domain (discussed below) to clamp FSH in place (Jiang et al. 2012). In LGR5, however, the C-terminal region does not form direct contact with either RSPO1 or RNF43; instead, the RNF43 PA domain binds to the other side of RSPO1, clamping the ligand in an analogous fashion.

Interestingly, each asymmetric unit reveals two copies of LGR5-RSPO1-RNF43 heterotrimers arranged around a twofold axis (Supplemental Fig. 2). The 2:2:2 complex is mediated between LGR5-LGR5 and LGR5-RNF43 interactions that involve the sides of the LRR solenoids, forming a back-to-back dimer resembling the TLR4-MD2 structure (Kim et al. 2007). Our gel filtration data, however, show primarily a 1:1:1 assembly ratio of LGR5, RSPO1, and RNF43, at least within the range of protein concentrations in our experiments. Due to uncertainties regarding the physiological relevance of the 2:2:2 complex, the following discussion focuses on the single LGR5-RSPO1-RNF43 complex.

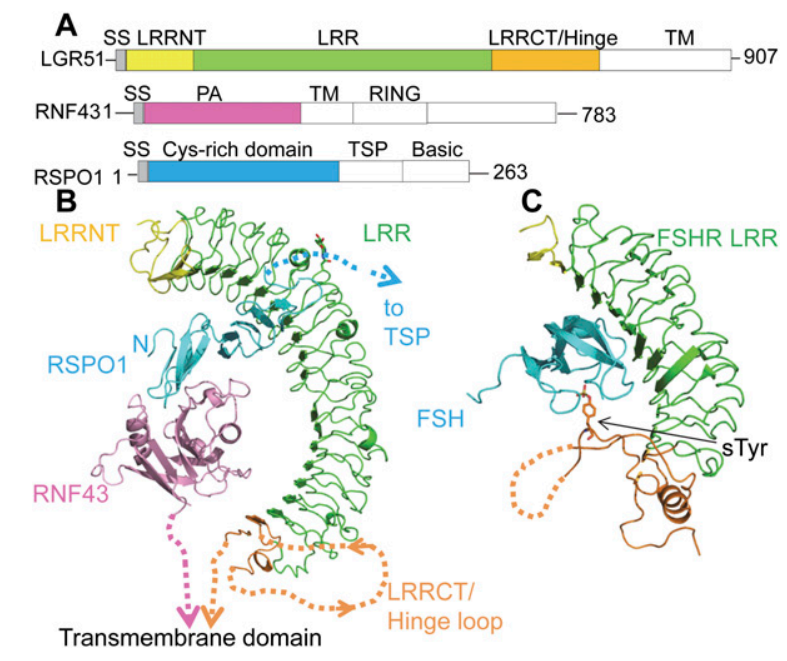

Figure 1. Crystal structure of the LGR5-RSPO1-RNF43 ternary complex. (A) Domain organization of human LGR5, RNF43, and RSPO1. The domains included for crystallization are colored. (LRRNT) LRR N-terminal domain; (LRRCT) LRR C-terminal domain; (TM) transmembrane. $(B)$ Ternary complex structure, color-coded as in $A$. The missing residues are depicted by dotted lines. N-linked glycosylation is shown as sticks. (Inset) Zoomed-in view of LRRCT/Hinge. (C) FSH-FSHR complex. The sulfated tyrosine is shown as sticks. Missing residues are depicted by dotted lines.
Divergence of the LGR5 scaffold from glycoprotein hormone receptors

Conforming to the general architecture of LRR domains (Bella et al. 2008), LGR5 contains an LRR N-terminal domain that caps the most $\mathrm{N}$-terminal LRR, followed by 17 LRRs that form a sleigh-like solenoid. Due to the large size of the LGR5 ECD, the entire solenoid adopts a slightly right-handed twist around LRR11 and LRR12. The large number of LRRs in LGR5 ECD encircles almost half a circle with a diameter of $\sim 80 \AA$, forming an arch that engulfs the entire vertical length of RSPO1 and RNF43 ( 50 A) under its concave face. Structure-based sequence alignment suggests that the 17 LRRs should be conserved from LGR4 to LGR6 and thus establishes the structural basis of functional equivalence between LGR4, LGR5, and LGR6.

The most C-terminal region of the LGR4-6 ECD contains a conserved hinge motif that differs from the one for glycoprotein hormone receptors (Hsu et al. 2000). For LGR4-6, this region consists of a helix with sequence PYAYQCC (residue 475-481) that caps the most C-terminal LRRs and is connected to another conserved sequence, GXFKPCE (residue 552-558), by a large flexible hinge loop whose electron density is lacking in our structure (residue 483-537). In FSHR, this so-called hinge loop is similarly present. Notably, a sulfur-modified Tyr (sTyr) on this loop serves as the secondary binding site for FSH (Supplemental Fig. 1). For LGR4-6, the absence of this critical sTyr, the lack of sequence conservation in the hinge loop residues, and the flexibility of the LRR C-terminal domain/hinge domain in the crystal structure altogether suggest that the membrane-proximal region is unlikely to interact with RNF43 or RSPO1 and diverges from the LGR1-3 hormone recognition mode. The difference in the hinge domain recognition step among LGR members rationalizes and potentially translates into the differential cytosolic signaling. Whereas the LGR1-3 hinge loop, upon hormone binding, is proposed to induce conformational changes in the transmembrane region and consequent G-protein coupling, the lack of hinge loop recognition in LGR4-6 may explain why G proteins are dispensable for LGR4-6 signaling (de Lau et al. 2011; Hao et al. 2012; Koo et al. 2012).

\section{Architecture of the RSPO1 CRD contains a head and a rod module}

The RSPO family appears only in vertebrates and selected invertebrates and does not share sequence similarities with other known Wnt signaling molecules (de Lau et al. 2012; Cruciat and Niehrs 2013). The RSPO1 N terminus contains a CRD commonly found in secreted proteins and transmembrane receptors. Overall, the structure can be characterized by a head module (from residue C40 to S78) (Fig. 2A, blue-green) and a rod module (from residue C79 to $\mathrm{C} 142$ ) (Fig. 2A, cyan) that were nearly perpendicular to one another. A ladder of eight strictly conserved disulfide bridges, numbered from $1-8$ from the $\mathrm{N}$ terminus to the $\mathrm{C}$ terminus, is identified and runs perpendicularly to the long axis of RSPO1. Dali server analysis identified the closest structural homology with CRD domains found in epidermal growth factor receptors (EGFRs; domain IV) and insulin receptors (IRs; domain II); nonetheless, the low $Z$-score of $\sim 4$ suggests that RSPO1 CRD adopts distinguishing features during evolution. 


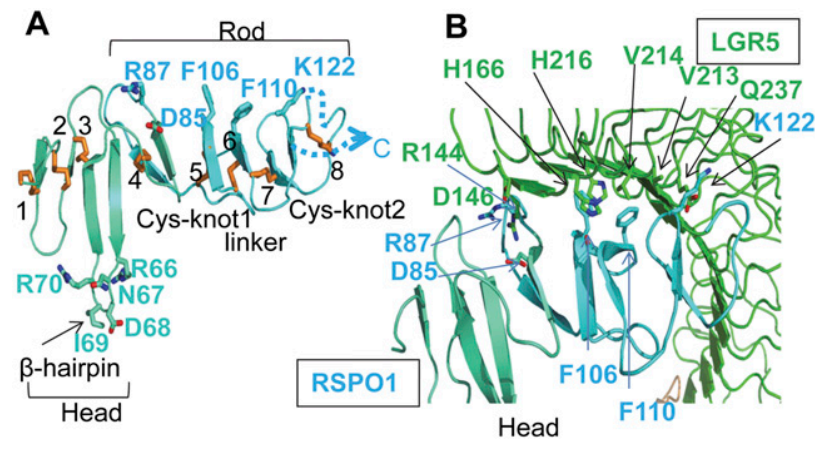

Figure 2. RSPO1 structure and interaction with LGR5. (A) The head module of RSPO1 is shown in blue-green, and the rod module is shown in cyan. The eight disulfide bridges are numbered and shown as sticks. The loop connected by disulfide 8 is disordered in the structure and is depicted as a dotted line. Selected hairpin loop residues and LGR5-interacting residues are shown as sticks. $(B)$ The interface between LGR5 and the RSPO1 rod module. Key interacting residues are shown in sticks.

Reflecting the conservation of all disulfide bridges in the CRD, missense mutations affecting the fourth and seventh disulfides compromise the secretion of RSPO4 and result in developmental defects of fingernails and toenails (anonychia) (Blaydon et al. 2006; Li et al. 2009; de Lau et al. 2012). In addition, RSPO1 homozygous mutation accounts for syndromic true hermaphroditism (Tomaselli et al. 2008); the mutation results in splicing variants lacking Ile95, and the register shift likely affects the nearby C79-C94 (disulfide 4) integrity.

Two separate regions form the major LGR5- and RNF43binding sites: a LGR5-binding rod module and a RNF43interacting head module (Fig. 2A). In the rod module, disulfide bridges 4 and 7 define two signature Cys-knot finger motifs that provide key recognition residues. These two finger motifs are further connected by a twisted linker whose rigidity is established by disulfides 5 and 6 and from which several aromatic residues (e.g., Phe106 and Phe110) emanate to form hydrophobic contact with LGR5. Together, these structural elements outline a shallow curve that embraces the concave face of LGR5. At the $\mathrm{N}$ terminus, the head module adopts a different structure than the rod module. A prominent, long $\beta$-hairpin protrusion (residue F61-L76) is anchored by the third disulfide to the main body of RSPO1 (Fig. 2A). As discussed below, this hairpin protrusion mediates major contact with the RNF43 PA domain.

\section{Conservation of RSPO1:LGR5 interface}

Consistent with previous findings, RSPO1 contacts the LGR5 N-terminal LRRs (de Lau et al. 2011); specifically, LRR4 to LRR9 cover the entire recognition interface (Fig. 2B), burying a total surface area of $\sim 2000 \AA^{2}$. The large surface comprises a small patch of hydrophilic and a large region of hydrophobic interactions (Fig. 2B; Supplemental Fig. 3; Supplemental Table 2); the involvement of a significant amount of hydrophobic interactions explains our observations that both RSPO1 and LGR5 have the tendency to aggregate when not in complex with one another and also the nanomolar affinity reported in literature (Carmon et al. 2011; de Lau et al. 2011; Glinka et al. 2011). The identified interface residues are conserved among all four RSPOs and LGR4-6, which explains the promiscuity in binding.

\section{RNF43 PA domain structure}

PA domains are commonly found in proteases as well as receptors such as transferrin receptors and plant-sorting receptors (Luo and Hofmann 2001), potentially function as ligand recognition motifs, and play regulatory roles. Perhaps underlying the critical roles for membrane trafficking in biology, transmembrane E3 ubiquitin ligases are found in various biological systems from plants to animals. For instance, in humans, RNF128, also called GRAIL, downregulates T-cell activity by using its extracellular PA domain to target certain receptors for endocytosis (Lineberry et al. 2008a,b; Whiting et al. 2011). Indeed, Dali analysis reveals that the RNF43 PA domain shares structural similarities with GRAIL/RNF128 (Z-score $=11.4)$, suggesting an evolutionarily conserved domain architecture. The RNF43 PA domain consists of seven $\beta$ strands (forming a twisted $\beta$ sheet) and three peripheral $\alpha$ helices. Of note, the curved $\beta 3$ outlines a shallow binding groove to which the RSPO1 $\beta$-hairpin protrusion binds (Fig. 3). Sequence similarity between RNF43 and ZNRF3 suggests that ZNRF3 shares similar structures and explains why the two proteins complement each other in in vivo studies (Koo, et al. 2012).

\section{RNF43 interaction with RSPO1}

The RSPO1:RNF43 interface is mediated between the RSPO1 $\beta$-hairpin protrusion (residue F61-L76) that fits in the shallow binding groove of RNF43. In contrast to the

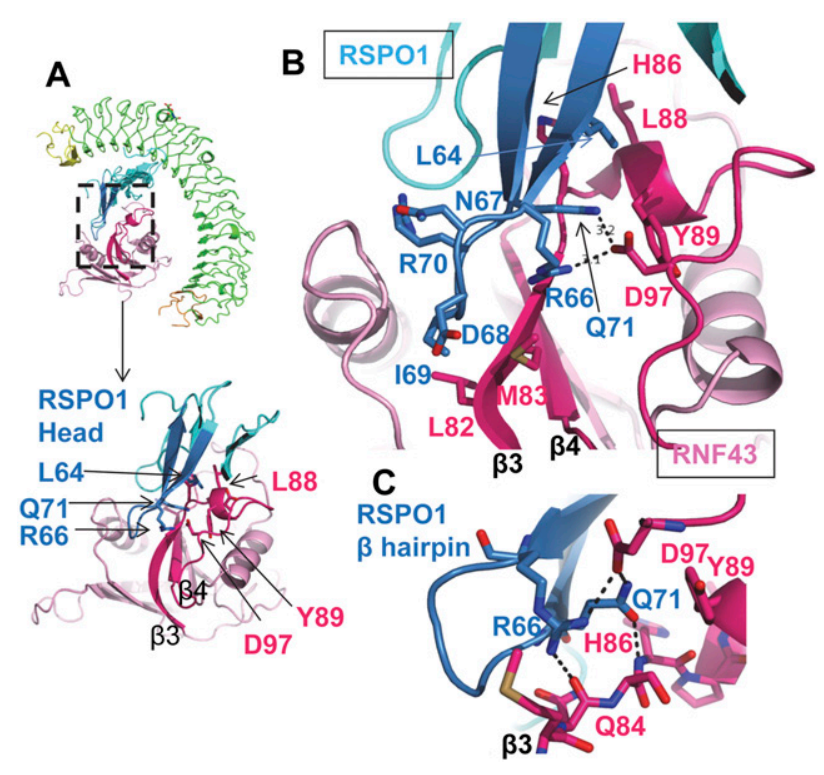

Figure 3. The head module of RSPO1 interaction with RNF43. For clarity, the RSPO1 $\beta$-hairpin protrusion is highlighted in marine, and key interacting loops and $\beta$ strands from RNF43 are colored in hot pink. (A) Miniature view showing RSPO1 hairpin insertion into the RNF43 groove. $(B)$ Magnified view, in the same orientation as $A$, of the boxed region in the ternary complex. RSPO1 R66/Q71 interaction with RNF43 D97 is shown as dashed lines. (C) Closer view of hydrogen bonding and salt bridge networks mediated between RSPO1 R66/Q71 and RNF43 Q84/H86/D97. 
largely hydrophobic nature of the RSPO1-LGR5 interface, a network of hydrophilic interactions forms the basis of this recognition and accounts for the micromolar affinity, as measured by isothermal titration calorimetry (ITC) (Fig. 5, below). Arg66 and Gln71 from the RSPO1 $\beta$ hairpin coordinate hydrogen bonds/salt bridges with Gln84, His86 (backbone), and Asp97 (side chain) from RNF43 and anchor the hairpin in the shallow binding groove (Fig. 3B,C; Supplemental Fig. 4; Supplemental Table 3). In addition, Leu64 from the hairpin forms a small region of hydrophobic contact with hydrophobic residues from RNF43 (His86, Leu88, and Tyr89) in a $3_{10}$ helix immediately following the $\beta 3$ strand. Taken together, the RSPO1 residues L64, R66, and Q71 are conserved across all four members of RSPO (except, in RSPO4, Leu is replaced by Ile) and thus represent a key recognition sequence. Moreover, the loop of the RSPO1 hairpin contains an Asn-Asp-Ile-Arg (residue 67-70) motif, with the Ile69 residue at the tip of the loop inserting into the RNF43 groove lined by bulky residues from RNF43 (e.g., Leu82 and Met83) (Fig. 3B). The fact that the loop does not fit perfectly into the groove suggests that a larger residue at position 69 could be tolerated. Indeed, in RSPO2 and RSPO3, Ile69 is replaced by the bulkier Met, which would make even more intimate contact with the pocket, in agreement with the degenerate binding of all four RSPOs with RNF43.

\section{Mapping disease mutations onto RSPO1 and RNF43}

The structure of the RSPO1:RNF43 interface provides a template for understanding disease mutations. Underlying the critical role of the RSPO1 $\beta$-hairpin protrusion in binding RNF43, a Q71R mutation in RSPO4 compromises its biological activities (Li et al. 2009). Mechanistically, substitution with Arg at the equivalent position in RSPO1 potentially leads to charge-charge repulsion with the neighboring R66 and induces steric clashes with RNF43 residues in the region (e.g., with Tyr89 side chain from RNF43), thus preventing RSPO $\beta$-hairpin interaction with RNF43 (Fig. 4B). Similarly, R60W in RSPO4, corresponding to R66 in RSPO1, also causes familial diseases (Khan et al. 2012); the hydrophilic-to-hydrophobic substitution would interrupt the hydrogen bonding/

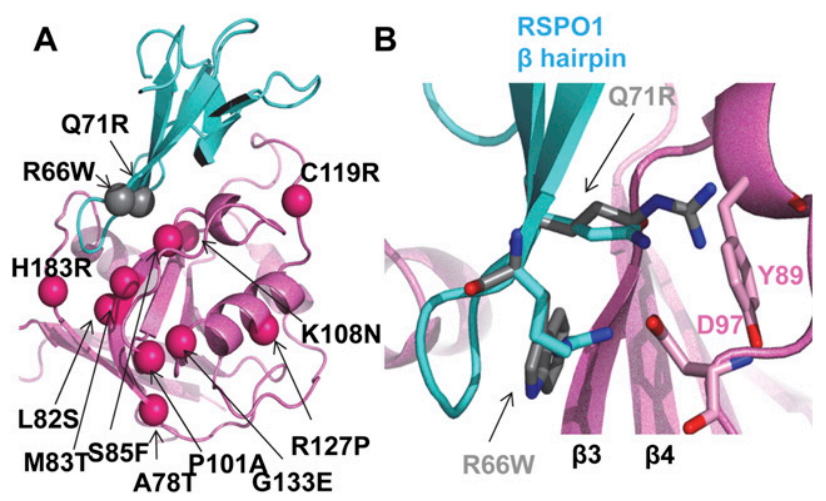

Figure 4. Selected known disease mutations mapped on RSPO1/ RNF43 structure. (A) Same view as in Figure 3A. Mutations are shown in ball presentations. (B) RSPO1 Q71R and R66W superimposed onto wild-type structure. salt bridge network with neighboring RNF43 residues Gln84 and D97.

In addition to RSPOs, many cancer mutations are identified in the tumor suppressor RNF43 PA domains (Koo et al. 2012; http://cancer.sanger.ac.uk/cosmic-search/s). Some map to the long, curved $\beta 3$ strand outlining the shallow RSPO1-binding groove (A78T, L82S, M83T, and S85F) (Fig. 4A), with the bulky S85F mutation probably the most disruptive of the protein core. Hao et al. (2012) pointed out a ZRNF3 P101A mutation that compromises binding with RSPO1. While this residue is not contacting any RSPO1 residues, the mutation likely disrupts the RNF43 loop connecting $\beta 3$ and $\beta 4$ held together by many hydrophobic side chains. Interruption of this core contact could cause register shift, resulting in a decreased affinity for RSPO1.

Currently, no mutations map to the RSPO1:LGR5 interface, and very few map onto the LGR5 ECD. The effect of LGR6 S299insGRS insertion (insertion of three amino acids, GRS, in S299) is more difficult to construe (Gong et al. 2012), as this region is not involved in RSPO1 binding. The insertion is located upstream of LRR11, at the center of the twisted LRR solenoid (Supplemental Fig. 1), suggesting that the intact LGR5 ECD curvature might play a role in signaling relay; i.e., forming proteinprotein interactions. Overall, the concentration of the disease mutations on the RSPO:ZRNF3/RNF43 side, instead of the RSPO:LGR side, likely supports that LGR4-6 play a primary role as the engagement receptors and that ZRNF3/ RNF43 play a primary role as the effector receptors, the latter more prone to modulation in signaling and diseases.

\section{Role of LGR5 in RNF43 recruitment}

The shallow and hydrophilic interface between RNF43 and RSPO1 could account for the 7-10 $\mu \mathrm{M}$ affinity between the two proteins as measured by ITC (Fig. 5). The weak interaction is further corroborated with the crystal structure, in which RNF43 and RSPO1 both show rather high $\mathrm{B}$ factors, implying thermal motion of polypeptides. Interestingly, when compared with RSPO1 alone, LGR5-RSPO1 displays $\sim 10$-fold higher affinity toward RNF43 $(\sim 0.5-1 \mu \mathrm{M})$, suggesting that addition of LGR5 stabilizes RNF43 in the final complex. Although there is no direct interaction between LGR5 and RNF43, LGR5 binding likely restricts the conformations of the thin structural elements of RSPO1, preparing RSPO1 entropically for more favorable binding toward RNF43; e.g., by reorienting the RSPO1 Gln71 and Arg66 to form hydrogen bonds/salt bridges with the RNF43 residues Gln84, His88, and Asp97. This, again, would be consistent with LGR5 serving as an engagement receptor that promotes the signaling complex assembly. Also in agreement with LGR5-enhanced RNF43 recruitment, previous function studies have shown that, although RSPO1 binds to ZNRF3 in a LGR4-independent manner, RSPO1-induced ZNRF3 membrane clearance is nonetheless LGR4-dependent (Hao et al. 2012).

\section{Implications of the LGR5-RSPO1-RNF43 complex structure in Wnt signaling}

The complexity of the Wnt regulatory system provides a rich resource for uncovering novel principles governing receptor-ligand interactions. Here, we provide the structural insight into RSPO recognition by LGR4-6 and RNF43/ 


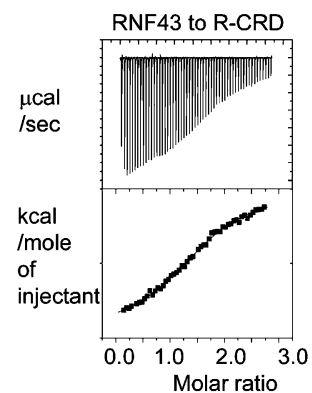

\section{$N=0.99$} $K \mathrm{~d}=10.70 \pm 0.44 \mu \mathrm{M}$ $\Delta H=-1.60 \pm 0.03 \mathrm{kcal} / \mathrm{mol}$ $\Delta S=18.2 \mathrm{cal} /\left(\mathrm{mol}^{*} \mathrm{~K}\right)$

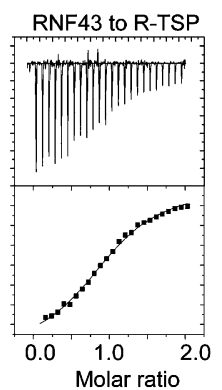

$N=1.01$ $K \mathrm{~d}=5.03 \pm 0.32 \mu \mathrm{M}$ $\Delta S=-1.70 \pm 0.04 \mathrm{kca} / \mathrm{m}$

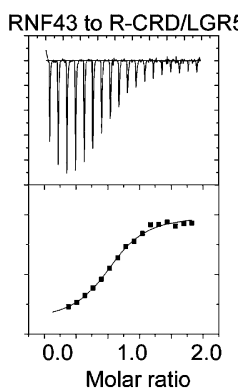

$N=1.01$ $K \mathrm{~d}=1.15 \pm 0.15 \mu \mathrm{M}$

$\Delta H=-3.28 \pm 0.09 \mathrm{kcal} / \mathrm{mo}$
RNF43 to R-TSP/LGR5

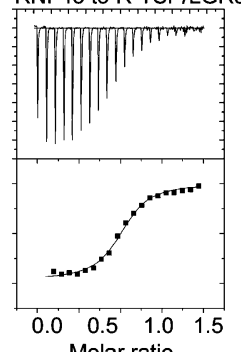

$N=0.99$

$K \mathrm{~d}=0.61 \pm 0.06 \mu \mathrm{M}$

$\Delta H=-3.86 \pm 0.06 \mathrm{kcal} / \mathrm{mol}$

$\Delta S=15.7 \mathrm{cal} /\left(\mathrm{mol}^{\star} \mathrm{K}\right)$

Figure 5. Calorimetric measurements of RNF43 interaction with RSPO1 alone or the RSPO1-LGR5 binary complex. (R-CRD) RSPO1 CRD only; (R-RSP) RSPO1 CRD+TSP.

ZNRF3, confirming that LGR4-6 and RNF43 function as RSPO receptors and showing two distinct chemical surfaces for simultaneous RSPO-LGR5 and RSPO-ZNRF3/RNF43 binding. Furthermore, despite the lack of direct interaction between LGR5 and RNF43, LGR5 increases the affinity of RNF43 to RSPO1. Conversely, RNF43 substitutes the hinge region in LGR4-6 as the potential "effector" domain for downstream signaling relay. Potentially, the weak interface between RNF43 and RSPO1 should be amenable to molecular competitions. As reported earlier, ZNRF3/RNF43 could be pulled down with Fz/LRP6 (Hao et al. 2012; Koo et al. 2012), suggesting that the PA domain could employ the same RSPO1-binding groove to engage other macromolecules (i.e., Fz/LRP6). The versatility of the RNF43:RSPO1 interface and the potential pleiotropism of RNF43 suggest potential applications for therapeutic design.

One key feature for RSPO signaling is that RSPOs synergize with not only the canonical Wnt/ $\beta$-catenin pathway, but also the noncanonical Wnt/PCP pathway. The common mechanistic basis for promoting both pathways has not been clear. To enhance the Wnt5a-mediated Wnt/ PCP pathway in Xenopus embryos, RSPO3 and RSPO4 were shown to bind with high affinity via their TSP domains to the proteoglycan Syndecan 4 receptor (SDC4) (Ohkawara et al. 2011). Interestingly, LGR4/5 also regulates Wnt/PCP signaling in Xenopus embryos (Glinka et al. 2011), with recent evidence suggesting that ZNRF3/RNF43 might play a role as well (Hao et al. 2012). These disparate results point to the possibility that LGR4-6, ZNRF3/RNF43, and SDC4 could coexist in the same complex. Our LGR5-RSPO1RNF43 model implies that, while the RSPO1 CRD binds to LGR5 and RNF43, the RSPO TSP domain would extend into an open space not occupied by other polypeptides (Fig. 1B) and is thus free to communicate with other molecules; i.e., SDC4. This model would place RSPOs at the organizing center to bring all three coreceptors together. In this regard, whether RSPOs feed into the Wnt/ $\beta$-catenin or Wnt/PCP pathway would then depend on the presence of SDC4 as well as on specific Wnt/Fz combinations.

In summary, the mechanistic details governing Wnt signaling regulation is only emerging. The LGR5-RSPO1RNF43 structure represents a step forward in understanding such a complex regulation. The central role of RSPOs in tethering coreceptors strongly suggests that they could be further explored as potential modulators for stem cell biology or regenerative medicine. Tris ( $\mathrm{pH}$ 8.0). X-ray diffraction data sets were collected at $100 \mathrm{~K}$ at the Life Science Collaborative Access Team (LS-CAT) beamline 21-ID-D (The Advanced Photon Source at Argonne National Laboratory). The structure was solved by SIRAS using iodine derivatives. Initial atomic models were built manually using $\mathrm{O}$ and COOT. A noncrystallographic twofold symmetry was maintained throughout the refinement process.

\section{Calorimetry}

Calorimetric titrations were implemented with a VP-ITC calorimeter (MicroCal) at $30^{\circ} \mathrm{C}$. The data were processed with MicroCal Origin 5.0 software.

Detailed experimental procedures are provided in the Supplemental Material.

\section{Accession number}

The structure factors and coordinates of the ternary complex have been deposited in the Protein Data Bank with accession code 4KNG.

\section{Acknowledgments}

We thank J. Brunzelle for support in data collection. X.H. is supported by NIH grant GM098259. The Structural Biology Facility is supported by the R.H. Lurie Comprehensive Cancer Center of Northwestern University.

\section{References}

Barker N, Clevers H. 2010. Leucine-rich repeat-containing G-proteincoupled receptors as markers of adult stem cells. Gastroenterology 138: 1681-1696.

Barker N, Rookmaaker MB, Kujala P, Ng A, Leushacke M, Snippert H, van de Wetering M, Tan S, Van Es JH, Huch M et al. 2012. Lgr5 ${ }^{+ \text {ve }}$ stem/progenitor cells contribute to nephron formation during kidney development. Cell Rep 2: 540-552.

Bella J, Hindle KL, McEwan PA, Lovell SC. 2008. The leucine-rich repeat structure. Cell Mol Life Sci 65: 2307-2333.

Binnerts ME, Kim KA, Bright JM, Patel SM, Tran K, Zhou M, Leung JM, Liu Y, Lomas WE 3rd, Dixon M, et al. 2007. R-spondin1 regulates Wnt signaling by inhibiting internalization of LRP6. Proc Nat1 Acad Sci 104: 14700-14705.

Blaydon DC, Ishii Y, O'Toole EA, Unsworth HC, Teh MT, Ruschendorf F, Sinclair C, Hopsu-Havu VK, Tidman N, Moss C, et al. 2006. The gene encoding R-spondin 4 (RSPO4), a secreted protein implicated in Wnt signaling, is mutated in inherited anonychia. Nat Genet 38: 1245-1247.

Carmon KS, Gong X, Lin Q, Thomas A, Liu Q. 2011. R-spondins function as ligands of the orphan receptors LGR4 and LGR5 to regulate Wnt/ $\beta$-catenin signaling. Proc Natl Acad Sci 108: 11452-11457.

Clevers H, Nusse R. 2012. Wnt/ $\beta$-catenin signaling and disease. Cell 149: 1192-1205. 


\section{Chen et al.}

Cruciat CM, Niehrs C. 2013. Secreted and transmembrane wnt inhibitors and activators. Cold Spring Harb Perspect Biol 5: a015081.

de Lau W, Barker N, Low TY, Koo BK, Li VS, Teunissen H, Kujala P, Haegebarth A, Peters PJ, van de Wetering M, et al. 2011. Lgr5 homologues associate with Wnt receptors and mediate R-spondin signalling. Nature 476: $293-297$.

de Lau WB, Snel B, Clevers HC. 2012. The R-spondin protein family. Genome Biol 13: 242.

Dukkipati A, Park HH, Waghray D, Fischer S, Garcia KC. 2008. BacMam system for high-level expression of recombinant soluble and membrane glycoproteins for structural studies. Protein Expr Purif 62: 160-170.

Glinka A, Dolde C, Kirsch N, Huang YL, Kazanskaya O, Ingelfinger D, Boutros M, Cruciat CM, Niehrs C. 2011. LGR4 and LGR5 are R-spondin receptors mediating $\mathrm{Wnt} / \beta$-catenin and $\mathrm{Wnt} / \mathrm{PCP}$ signalling. $E M B O$ Rep 12: 1055-1061.

Gong X, Carmon KS, Lin Q, Thomas A, Yi J, Liu Q. 2012. LGR6 is a high affinity receptor of R-spondins and potentially functions as a tumor suppressor. PLOS ONE 7: e37137.

Hao HX, Xie Y, Zhang Y, Charlat O, Oster E, Avello M, Lei H, Mickanin C, Liu D, Ruffner H, et al. 2012. ZNRF3 promotes Wnt receptor turnover in an R-spondin-sensitive manner. Nature 485: 195-200.

Hsu SY, Kudo M, Chen T, Nakabayashi K, Bhalla A, van der Spek PJ, van Duin M, Hsueh AJ. 2000. The three subfamilies of leucine-rich repeat-containing G protein-coupled receptors (LGR): Identification of LGR6 and LGR7 and the signaling mechanism for LGR7. Mol Endocrinol 14: 1257-1271.

Huch M, Dorrell C, Boj SF, van Es JH, Li VS, van de Wetering M, Sato T, Hamer K, Sasaki N, Finegold MJ, et al. 2013. In vitro expansion of single $\mathrm{Lgr5}^{+}$liver stem cells induced by Wnt-driven regeneration. Nature 494: $247-250$.

Janda CY, Waghray D, Levin AM, Thomas C, Garcia KC. 2012. Structural basis of Wnt recognition by Frizzled. Science 337: 59-64.

Jiang X, Liu H, Chen X, Chen PH, Fischer D, Sriraman V, Yu HN, Arkinstall S, He X. 2012. Structure of follicle-stimulating hormone in complex with the entire ectodomain of its receptor. PNAS 109: 12491-12496.

Kamata T, Katsube K, Michikawa M, Yamada M, Takada S, Mizusawa H. 2004. R-spondin, a novel gene with thrombospondin type 1 domain, was expressed in the dorsal neural tube and affected in Wnts mutants. Biochim Biophys Acta 1676: 51-62.

Kazanskaya O, Glinka A, del Barco Barrantes I, Stannek P, Niehrs C, Wu W. 2004. R-Spondin 2 is a secreted activator of Wnt $/ \beta$-catenin signaling and is required for Xenopus myogenesis. Dev Cell 7: 525-534.

Khan TN, Klar J, Nawaz S, Jameel M, Tariq M, Malik NA, Baig SM, Dahl N. 2012. Novel missense mutation in the RSPO4 gene in congenital hyponychia and evidence for a polymorphic initiation codon (p.M1I). BMC Med Genet 13: 120.

Kim HM, Park BS, Kim JI, Kim SE, Lee J, Oh SC, Enkhbayar P, Matsushima N, Lee H, Yoo OJ, et al. 2007. Crystal structure of the TLR4-MD-2 complex with bound endotoxin antagonist Eritoran. Cell 130: 906-917.

Kong RC, Shilling PJ, Lobb DK, Gooley PR, Bathgate RA. 2010. Membrane receptors: Structure and function of the relaxin family peptide receptors. Mol Cell Endocrinol 320: 1-15.

Koo BK, Spit M, Jordens I, Low TY, Stange DE, van de Wetering M, van Es JH, Mohammed S, Heck AJ, Maurice MM, et al. 2012. Tumour suppressor RNF43 is a stem-cell E3 ligase that induces endocytosis of Wnt receptors. Nature 488: 665-669.

Li SJ, Yen TY, Endo Y, Klauzinska M, Baljinnyam B, Macher B, Callahan R, Rubin JS. 2009. Loss-of-function point mutations and two-furin domain derivatives provide insights about R-spondin2 structure and function. Cell Signal 21: 916-925.

Lineberry N, Su L, Soares L, Fathman CG. 2008a. The single subunit transmembrane E3 ligase gene related to anergy in lymphocytes (GRAIL) captures and then ubiquitinates transmembrane proteins across the cell membrane. J Biol Chem 283: 28497-28505.

Lineberry NB, Su LL, Lin JT, Coffey GP, Seroogy CM, Fathman CG. 2008b. Cutting edge: The transmembrane E3 ligase GRAIL ubiquitinates the costimulatory molecule CD40 ligand during the induction of T cell anergy. J Immunol 181: 1622-1626.

Luo X, Hofmann K. 2001. The protease-associated domain: A homology domain associated with multiple classes of proteases. Trends Biochem Sci 26: 147-148.
Niehrs C. 2012. The complex world of WNT receptor signalling. Nat Rev Mol Cell Biol 13: 767-779.

Ohkawara B, Glinka A, Niehrs C. 2011. Rspo3 binds syndecan 4 and induces Wnt/PCP signaling via clathrin-mediated endocytosis to promote morphogenesis. Dev Cell 20: 303-314.

Parma P, Radi O, Vidal V, Chaboissier MC, Dellambra E, Valentini S, Guerra L, Schedl A, Camerino G. 2006. R-spondinl is essential in sex determination, skin differentiation and malignancy. Nat Genet 38: 1304-1309.

Ruffner H, Sprunger J, Charlat O, Leighton-Davies J, Grosshans B, Salathe A, Zietzling S, Beck V, Therier M, Isken A, et al. 2012. R-spondin potentiates $\mathrm{Wnt} / \beta$-catenin signaling through orphan receptors LGR4 and LGR5. PLOS ONE 7: e40976.

Snippert HJ, Haegebarth A, Kasper M, Jaks V, van Es JH, Barker N, van de Wetering M, van den Born M, Begthel H, Vries RG, et al. 2010. Lgr6 marks stem cells in the hair follicle that generate all cell lineages of the skin. Science 327: 1385-1389.

Tomaselli S, Megiorni F, De Bernardo C, Felici A, Marrocco G, Maggiulli G, Grammatico B, Remotti D, Saccucci P, Valentini F, et al. 2008. Syndromic true hermaphroditism due to an R-spondin1 (RSPO1) homozygous mutation. Hum Mutat 29: 220-226.

Wei Q, Yokota C, Semenov MV, Doble B, Woodgett J, He X. 2007. R-spondin1 is a high affinity ligand for LRP6 and induces LRP6 phosphorylation and $\beta$-catenin signaling. I Biol Chem 282: 1590315911.

Whiting CC, Su LL, Lin JT, Fathman CG. 2011. GRAIL: A unique mediator of CD4 T-lymphocyte unresponsiveness. FEBS J 278: 47-58. 


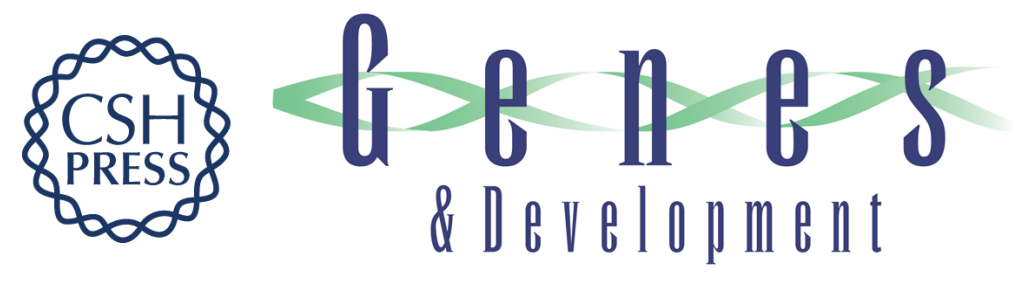

\section{The structural basis of R-spondin recognition by LGR5 and RNF43}

Po-Han Chen, Xiaoyan Chen, Zhenghong Lin, et al.

Genes Dev. 2013, 27: originally published online June 11, 2013

Access the most recent version at doi:10.1101/gad.219915.113

\footnotetext{
Supplemental http://genesdev.cshlp.org/content/suppl/2013/06/04/gad.219915.113.DC1

Material

Related Content Structural basis for $R$-spondin recognition by LGR4/5/6 receptors

Dongli Wang, Binlu Huang, Senyan Zhang, et al.

Genes Dev. June, 2013 27: 1339-1344 R-Spondin Co-Receptors

Annalisa M. VanHook

Sci. Signal. July , 2013 6: ec151

References This article cites 36 articles, 9 of which can be accessed free at:

http://genesdev.cshlp.org/content/27/12/1345.full.html\#ref-list-1

Articles cited in:

http://genesdev.cshlp.org/content/27/12/1345.full.html\#related-urls

License

Email Alerting
Service $\begin{aligned} & \text { Receive free email alerts when new articles cite this article - sign up in the box at the top } \\ & \text { right corner of the article or click here. }\end{aligned}$
}

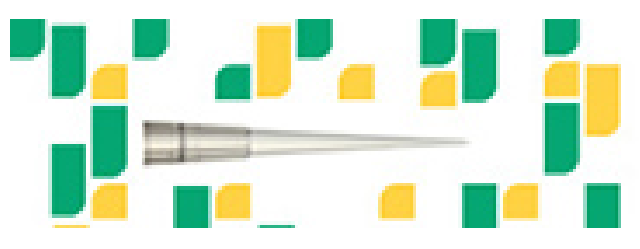

Focused on your science. 